\title{
X-ray phase contrast imaging with polychromatic sources and the concept of effective energy
}

\author{
Peter R.T. Munro \\ Optical + Biomedical Engineering Laboratory and Centre for Microscopy, Characterisation and Analysis, \\ The University of Western Australia, Crawley, Western Australia 6009, Australia*
}

Alessandro Olivo

Department of Medical Physics and Bioengineering,

University College London, London WC1E 6BT, UK

(Dated: June 25, 2013)

\begin{abstract}
Grating based quantitative polychromatic x-ray phase imaging is currently a very active area of research. It has already been shown that, in such systems, the retrieved differential phase depends upon the spectral properties of the source, gratings, detector and sample. In this paper, we show that the retrieved sample absorption also depends upon the spectral properties of the gratings. Further, we compare the spectral dependence of both retrieved phase and absorption for the grating interferometer and coded aperture techniques. These results enable us to conclude that in both cases, quantitative phase imaging systems cannot be described by an effective energy which is independent of the sample. This has important implications for applications where an absolute measure of phase is important and in tomography.
\end{abstract}

\section{INTRODUCTION}

There are now a number of groups around the world developing grating based $\mathrm{x}$-ray phase contrast imaging (XPCI) systems which employ non micro-focus laboratory sources [1-4]. Grating based techniques seek to obtain quantitative phase gradient and absorption images of a sample. Apart from providing planar images of much improved detail and clarity, quantitative XPCI is also an essential step in attaining three dimensional tomographic images. The development of quantitative XPCI systems employing non micro-focus laboratory sources has been driven by the desire to apply the technique away from synchrotrons and in applications where the long exposure times imposed by micro-focus sources are not practical.

Quantitative, polychromatic, differential phase imaging using a conventional x-ray source was demonstrated using a grating interferometer by Pfeiffer et. al [1]. The authors of this paper deduced the "mean energy of the effective x-ray spectrum", using material property data in order to quantitatively compare measured phase values with the tabulated values. Subsequent publications employing a grating interferometer [5] and the coded aperture technique [6] have shown experimentally that the concept of a mean energy depends upon whether phase or absorption is considered and that these two are, in general, different. This has been studied in detail for the grating interferometry case by Chabior et. al [7] who showed that the measured phase gradient depends upon the spectral properties of the gratings, detector, source and sample. Crucially, it was noted in this timely work that sample absorption affects the measured value of phase gradient. In this paper we derive equations con-

\footnotetext{
* peter.munro@uwa.edu.au
}

sistent with those of Chabior et. al [7] which, however, explicitly take into account the spectral dependence of the apertures. This is done in order to show that both the measured absorption and phase are influenced by the spectral properties of the imaging system. Further, the derived equations show that the spectral dependencies of the coded aperture and grating systems differ. We also show experimental results for weakly and strongly absorbing materials to verify the derived theoretical results.

\section{THEORETICAL RESULTS}

Both Talbot(-Lau) interferometry and coded aperture XPCI employ periodic structures to generate and sense periodic x-ray intensity patterns. Phase information is encoded in perturbations to the position of such patterns and absorption information in the average intensity. The Talbot(-Lau) interferometer [1, 4] employs a phase grating to form Lohmann fringes at particular distances downstream of the grating [8], thus eliminating the deleterious effects of diffraction. The method requires a source with fractional spectral width not exceeding $10 \%$ [1] and a source grating [1] or a grooved source target [4] to increase the source spatial coherence, as shown in Fig. 1. An alternative to Talbot(-Lau) interferometry, called coded aperture XPCI, employs an absorbing grating prior to the sample and is also sketched in Fig. 1. The transmitting regions of this grating are made sufficiently wide such that the collimated beams are perturbed only weakly by diffraction, prior to being incident upon the detector grating. In this configuration, the effects of low temporal and spatial coherence which arise from using a laboratory source are actually beneficial in smoothing the profile of the beam incident upon the detector aperture 
[9]. A sample placed downstream of the sample grating allows refracted x-rays to be efficiently detected using the principle of pixel edge illumination $[2,10]$. This technique decouples the system's sensitivity from the width of the transmitting region of the grating and also from the pitch of the gratings [6].

For both the grating and coded aperture techniques, the displacement and average intensity of fringes and/or $\mathrm{x}$-ray beams is sensed by using a grating immediately before the detector, represented by $\mathrm{G}_{2}$ in Fig. 1 . The detector grating can be parallel to the fringes or rotated about the optical axis by a slight angle, resulting in the Moiré configuration [11]. In the parallel case a technique called phase stepping is employed whereby the intensity of each pixel in the detector is recorded for a sequence of lateral positions of the detector grating. Becaus phase stepping is employed, the pixel dimension, $P$, and $p_{2}$ need not be equal, and in general, $P>p_{2}[1,3,4]$. Phase stepping is not employed in the case of coded aperture XPCI, instead images for two equal and opposite values of $\Delta P$ are acquired, from which the phase is extracted [6]. In contrast to the Talbot(-Lau) setup, coded aperture XPCI requires $p_{2}$ to be equal to an integer multiple of $P$, most commonly having $p_{2}=P$. Furthermore, each transmitting region of $\mathrm{G}_{2}$ must be contained within a pixel.

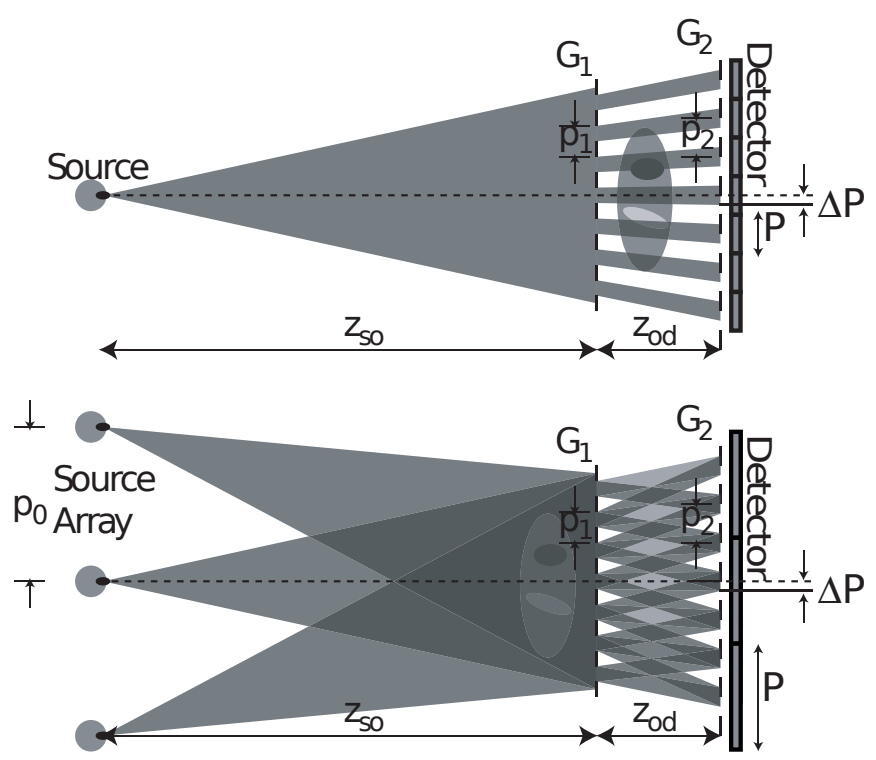

FIG. 1. Arrangements for the coded aperture (top) and grating interferometry (lower) systems with samples included. For clarity, these diagrams are not scaled in accordance with the prevailing practical implementations, for example, the pitches of $G_{1}$ and $G_{2}$ in the lower system are typically of the order of 5 to 20 times smaller than those of the upper system.

Irrespective of which system in Fig. 1 is employed, in the absence of a sample, a periodic intensity pattern is projected by grating $\mathrm{G}_{1}$ onto $\mathrm{G}_{2}$ which may be described by an energy density function as [11]:

$$
\begin{aligned}
I(x, \lambda) & \approx \exp (-2 \mu(x / M, \lambda))\left(a_{0}^{\mathrm{f}}(\lambda)\right. \\
& \left.+\sum_{n} b_{n}^{\mathrm{f}}(\lambda) \cos \left(2 \pi n\left(x+z_{\text {od }} \phi_{x}(x / M, \lambda)\right) / p_{2}\right)\right)
\end{aligned}
$$

where the superscript $\mathrm{f}$ denotes fringe, $\phi_{x}(x, \lambda)=$ $(\partial / \partial x) \int_{\mathcal{O}} \delta(x, z, \lambda) \mathrm{d} z, \mu(x, \lambda)=k \int_{\mathcal{O}} \beta(x, z, \lambda) \mathrm{d} z, \delta$ is the refractive index decrement, $\beta$ the imaginary part of the refractive index, $k=2 \pi / \lambda$ is the wave number, $\lambda$ is the wavelength and integration taken is over the object along the direction of wave propagation. Note that $\phi_{x}$ is strictly the refraction angle but since this is proportional to phase gradient we still refer hereafter to it as phase gradient. Note also that $\mu$ is different to the attenuation coefficient usually employed in x-ray radiography. We note that despite the depicted locations of the samples in the systems of Fig. 4, Eq. (1) is only strictly valid when the sample is in the plane of $G_{1}$. This approximation has however been shown to be reasonable in practice $[7,12,13]$ and does not affect the conclusions of this paper. Furthermore, in arriving at Eq. (1) we have assumed that the sample satisfies the projection approximation, meaning that the sample is sufficiently thin and that inhomogeneities are large compared with the wavelength [14]. In this context, one criterion for being sufficiently thin is that the thickness is much less than $\mathrm{z}_{\text {so }}[15]$ although Paganin [16] presents a thorough discussion of this subject. Furthermore, whilst the unperturbed fringe pattern may be calculated using any means, prior to decomposing onto a sinusoidal basis, we have assumed that the first order stationary phase approximation may be employed in modelling how the sample perturbs the fringe pattern, as is made by nearly all researchers in the field that we are aware of. Next we introduce the detector grating with an energy transmission function which is also described by a function of period $p_{2}$ and can thus be described as:

$$
T(x, \Delta P, \lambda)=a_{0}^{\mathrm{a}}(\lambda)+\sum_{n} b_{n}^{\mathrm{a}}(\lambda) \cos \left(2 \pi n(x-\Delta P) / p_{2}\right)(2)
$$

where $\Delta P$ represents the position of the grating. Note that the dependence of $a_{0}^{f / a}$ and $b_{n}^{f / a}$ upon wavelength embodies the spectral properties of the gratings which lead to partial transmission by absorption gratings and non-ideal phase modulation by phase gratings. Neglecting diffraction between the detector grating and the detector, the signal in pixel $j$ is then obtained as:

$$
I_{j}(\Delta P)=\frac{1}{p_{2}} \iint_{(j-1 / 2) p_{2}}^{(j+1 / 2) p_{2}} T(x, \Delta P, \lambda) I(x, \lambda) \sigma(\lambda) \mathrm{d} x \mathrm{~d} \lambda
$$

where $\sigma(\lambda)$ is an effective source spectrum combining the source spectrum, detector response and attenuation by air and the grating substrate, normalised such that $\int \sigma(\lambda) \mathrm{d} \lambda=1$. Note that for simplicity we have chosen $P=p_{2}$ for Talbot(-Lau) case which is not necessarily the case in practice, however, the expressions which we derive later to elucidate the spectral dependence of the system remain general with respect to the choice of pixel size. Note also that integration with respect to $\lambda$ is taken over the source spectrum, as is the case in all remaining integrations over $\lambda$. Substituting Eqs. (1) and (2) into Eq. 
(3) and assuming that $\mu$ and $\phi_{x}$ remain approximately constant within one period of the sample grating gives

$$
I_{j}(\Delta P) \approx a_{j}^{0}+a_{j}^{1} \cos \left(2 \pi \Delta P / p_{2}\right)-a_{j}^{2} \sin \left(2 \pi \Delta P / p_{2}\right)
$$

where

$$
\begin{aligned}
a_{j}^{0}= & \int \exp \left(-2 \mu\left(j p_{2} / M, \lambda\right)\right) a_{0}^{\mathrm{a}}(\lambda) a_{0}^{\mathrm{f}}(\lambda) \sigma(\lambda) \mathrm{d} \lambda \\
a_{j}^{1}= & \frac{1}{2} \int \exp \left(-2 \mu\left(j p_{2} / M, \lambda\right)\right) b_{1}^{\mathrm{a}}(\lambda) b_{1}^{\mathrm{f}}(\lambda) \\
& \cdot \cos \left(2 \pi z_{o d} \phi_{x}\left(j p_{2} / M, \lambda\right) / p_{2}\right) \sigma(\lambda) \mathrm{d} \lambda \\
a_{j}^{2}= & \left.\frac{1}{2} \int \exp \left(-2 \mu\left(j p_{2} / M, \lambda\right)\right) b_{1}^{\mathrm{a}}(\lambda) b_{1}^{\mathrm{f}}(\lambda)\right) \\
& \cdot \sin \left(2 \pi z_{o d} \phi_{x}\left(j p_{2} / M, \lambda\right) / p_{2}\right) \sigma(\lambda) \mathrm{d} \lambda
\end{aligned}
$$

When phase stepping is employed, a least squares solution for $a_{j}^{0}, a_{j}^{1}$ and $a_{j}^{2}$ may be found by taking measurements for $N$ different values of $\Delta P$ forming the vector $\Delta \mathbf{P}=\left(\Delta P_{1}, \Delta P_{2}, \ldots, \Delta P_{N}\right)[17]$. In particular, $a_{j}^{0}, a_{j}^{1}$ and $a_{j}^{2}$ may be found from experimental measurements as the result of a matrix equation:

$$
\left[\begin{array}{lll}
a_{j}^{0} & a_{j}^{1} & a_{j}^{2}
\end{array}\right]^{T}=\mathbf{A}^{-1}(\boldsymbol{\Delta} \mathbf{P}) \mathbf{B}\left(I_{j}(\boldsymbol{\Delta} \mathbf{P}), \boldsymbol{\Delta} \mathbf{P}\right)
$$

where $\mathbf{A}$ is a $3 \times 3$ matrix which is a function of $\boldsymbol{\Delta} \mathbf{P}$ only and $\mathbf{B}$ is a $3 \times 1$ vector which is a function of $I_{j}(\Delta \mathbf{P})$ and $\boldsymbol{\Delta} \mathbf{P}$ only [17]. This shows that the resulting values of $a_{j}^{0}, a_{j}^{1}$ and $a_{j}^{2}$ have no explicit dependence upon $\lambda$. The mean sample absorption, $\bar{\mu}\left(j p_{2} / M\right)$, can be found from $a_{j}^{0}$ as

$$
\exp \left(-2 \bar{\mu}\left(j p_{2} / M\right)\right)=\frac{a_{j}^{0}}{\int a_{0}^{\mathrm{a}}(\lambda) a_{0}^{\mathrm{f}}(\lambda) \sigma(\lambda) \mathrm{d} \lambda}
$$

where the denominator in Eq. (9) may be found by acquiring an image in the absence of a sample. This result holds for both grating and coded aperture XPCI and shows that the absorption image retrieved using both methods differs from that obtained using conventional absorption radiography. Coded aperture XPCI, however, reduces this discrepancy by using apertures $\mathrm{G}_{1}$ and $\mathrm{G}_{2}$ with opaque regions which are approximately fully absorbing throughout the spectrum. This is possible because the larger scale gratings are able to be made with thicker absorbing material.

Extraction of the phase gradient is slightly more complicated. In the monochromatic case, $\phi_{x}$ is extracted according to $\tan \left(2 \pi z_{o d} \phi_{x}\left(j p_{2} / M\right) / p_{2}\right)=-a_{j}^{2} / a_{j}^{1}$. In the polychromatic case, assuming small $\phi_{x}$ such as is encountered in the experimental results in this paper, this procedure will extract an average phase gradient given by:

$$
\overline{\phi_{x}}\left(j p_{2} / M\right) \approx \int W_{j}(\lambda) \phi_{x}\left(j p_{2} / M, \lambda\right) \mathrm{d} \lambda
$$

where

$W_{j}(\lambda)=W_{j}^{p s}(\lambda)=\frac{\exp \left(-2 \mu\left(j p_{2} / M, \lambda\right)\right) b_{1}^{\mathrm{a}}(\lambda) b_{1}^{\mathrm{f}}(\lambda) \sigma(\lambda)}{\int \exp \left(-2 \mu\left(j p_{2} / M, \lambda\right)\right) b_{1}^{\mathrm{a}}(\lambda) b_{1}^{\mathrm{f}}(\lambda) \sigma(\lambda) \mathrm{d} \lambda}$

This result is in agreement with Chabior et. al [7] who, however, do not make the assumption of small phase gradients and use a visibility function in the place of the product $b_{1}^{\mathrm{a}}(\lambda) b_{1}^{\mathrm{f}}(\lambda)$. The coded aperture technique doesn't employ phase stepping as only two images are acquired. In the formalism of this paper, two images are acquired corresponding to $I_{j}\left( \pm p_{2} / 4\right)$ which gives

$$
I_{j}\left(p_{2} / 4\right)=a_{j}^{0}-a_{j}^{2} \text { and } I_{j}\left(-p_{2} / 4\right)=a_{j}^{0}+a_{j}^{2}
$$

The mean absorption may then be found using Eq. (9) since $a_{j}^{0}=\left(I_{j}\left(p_{2} / 4\right)+I_{j}\left(-p_{2} / 4\right)\right) / 2$. The phase gradient is extracted by forming

$$
\frac{I_{j}\left(-p_{2} / 4\right)-I_{j}\left(p_{2} / 4\right)}{I_{j}\left(-p_{2} / 4\right)+I_{j}\left(p_{2} / 4\right)}=\frac{a_{j}^{2}}{a_{j}^{0}}
$$

which reveals that in the case of coded aperture XPCI, the mean phase gradient is described by Eq. (10) but with $W_{j}$ defined by

$$
\begin{aligned}
& W_{j}(\lambda)=W_{j}^{c a}(\lambda)= \\
& \frac{\hat{a}_{0}^{a} \hat{a}_{0}^{f}}{\hat{b}_{1}^{a} \hat{b}_{1}^{f}} \frac{\exp \left(-2 \mu\left(j p_{2} / M, \lambda\right)\right) b_{1}^{\mathrm{a}}(\lambda) b_{1}^{\mathrm{f}}(\lambda) \sigma(\lambda)}{\int \exp \left(-2 \mu\left(j p_{2} / M, \lambda\right)\right) a_{0}^{\mathrm{a}}(\lambda) a_{0}^{\mathrm{f}}(\lambda) \sigma(\lambda) \mathrm{d} \lambda}
\end{aligned}
$$

where $\hat{a}_{0}^{a / f}$ and $\hat{b}_{1}^{a / f}$ are the Fourier coefficients in the case that the apertures are completely absorbing and thus have no spectral dependence. The weighted average expression in Eq. (10) in conjunction with Eqs. (11) and (14) formalises an intuitive result and has some important implications for the interpretation of measurements made by such a system. Firstly, dispersive analyser and/or sample gratings will strongly influence the measured differential phase. Secondly, the absorption properties of a sample directly affect the measurement of differential phase. Finally, the effective source spectrum and detector characteristics strongly affect measurement of differential phase.

The concept of effective energy or mean energy is frequently used when testing polychromatic XPCI systems. In absorption radiography it is routinely said that a combination of system, $\sigma(\lambda)$, and sample, $\mu(x, \lambda)$, has an effective wavelength $\lambda_{\text {eff }}^{\mu}$ satisfying $\exp \left(-2 \mu\left(x, \lambda_{\text {eff }}^{\mu}\right)\right)=$ $\int \exp (-2 \mu(x, \lambda)) \sigma(\lambda) \mathrm{d} \lambda[18]$. Since $\mu(x, \lambda)$ depends upon the thickness of the sample, in general, the effective wavelength also depends upon the thickness of the sample. In the case of a sample with low absorption this reduces to the more convenient expression: $\mu\left(x, \lambda_{\text {eff }}^{\mu}\right)=\int \mu(x, \lambda) \sigma(\lambda) \mathrm{d} \lambda$.

We can use a similar argument for differential phase imaging. In particular, we define an effective wavelength $\lambda_{\text {eff }}^{\phi}$ satisfying $\phi_{x}\left(x, \lambda_{\text {eff }}^{\phi}\right)=\overline{\phi_{x}}(x)$ which, from inspection of Eq. (10), will, in general, not equal $\lambda_{\text {eff. Furthermore, }}^{\mu}$ a sample will, in general, have a range of effective energies due to variable absorption throughout a sample. In the special case of negligible absorption in the sample and non-dispersive gratings, we find $\lambda_{\text {eff }}^{\phi}$ satisfies the intuitive relationship: $\phi_{x}\left(x, \lambda_{\text {eff }}^{\phi}\right)=\int \phi_{x}(x, \lambda) \sigma(\lambda) \mathrm{d} \lambda$. Note that 
even in the case of low absorption, the effective wavelengths $\lambda_{\text {eff }}^{\phi}$ and $\lambda_{\text {eff }}^{\mu}$ will differ since $\beta(\lambda)$ and $\delta(\lambda)$ depend differently on $\lambda$. This has been observed previously $[1,5,6]$ but was not explained in those publications.

\section{EXPERIMENTAL AND SIMULATED RESULTS}

We now verify the theoretical results by comparing simulated and experimental data. The experiment is described in detail elsewhere [6] however, we give a brief description of it here. We employed a coded aperture system as depicted in Fig. 1 with $z_{s o}=1.6 \mathrm{~m}, z_{\text {od }}=0.4 \mathrm{~m}$, $p_{2}=85 \mu \mathrm{m}$, a source focal spot FWHM of $60 \mu \mathrm{m}$ and masks composed of $20 \mu \mathrm{m}$ thick gold. A Rigaku $007 \mathrm{HF}$ source operated at $35 \mathrm{kV} / 25 \mathrm{~mA}$ (mean energy approximately $18 \mathrm{keV}$ ) with a rotating Mo target was used. A titanium wire of radius $130 \mu \mathrm{m}$ was employed for the comparison between theory and experiment. We simulated the polychromatic phase and absorption images of a titanium wire using a calculated effective source spectrum including the effect of the aperture's graphite substrate and propagation in air [19], and tabulated values for $\delta(\lambda)$ and $\beta(\lambda)$ [20]. The simulation proceeded by first calculating $a_{0}^{f / a}$ and $b_{n}^{f / a}$, using only geometrical optics, taking into account the source FWHM and spectrum and tabulated refractive index data for gold [20]. Then the $W_{j}^{c a}(\lambda)$ were calculated using Eq. (14), for each discrete point, and therefore thickness, along the cross-section of the titanium wire and for each wavelength in the sampled spectrum. This enabled $\bar{\mu}(x)$ and $\overline{\phi_{x}}(x)$ to be calculate using Eqs. (9) and (10) respectively. Fig. 2 demonstrates a close match between the experimentally obtained, polychromatic, $\overline{\phi_{x}}$ and that obtained by Eq. (10). Note also that these two differ appreciably from $\bar{\phi}_{x}$ obtained by using $\delta$ at the mean energy of the spectrum. This plot illustrates an important result of Eq. (10), that polychromatic phase imaging can result in a differential phase gradient with an incorrect shape. The plot of $\overline{\phi_{x}}$ at the spectrum mean energy possesses the correct shape, however, $\overline{\phi_{x}}$ obtained by Eq. (10) differs by more than a constant scaling factor due to varying sample thickness as discussed in detail below.

Having calculated $\bar{\mu}(x)$ and $\overline{\phi_{x}}(x)$, the mean values of $\bar{\beta}(x)$ and $\bar{\delta}(x)$ were found since the shape of the sample was known. The effective energies, at each point along the wire cross section, were found by interpolating the tabulated refractive index values [20] such that $\beta\left(x, \lambda_{\text {eff }}^{\mu}\right)=\bar{\beta}(x)$ and $\delta\left(x, \lambda_{\text {eff }}^{\phi}\right)=\bar{\delta}(x)$. Fig. 3 shows plots of the calculated effective energies for phase and absorption across the titanium sample, as a function of sample thickness, as well as the mean energy of the spectrum. The plots demonstrate how the effective energies for phase and absorption are, in general, not equal and actually vary with sample thickness. Both effective energies tend to increase with increasing sample thickness. In both cases this is due to beam hardening as the higher

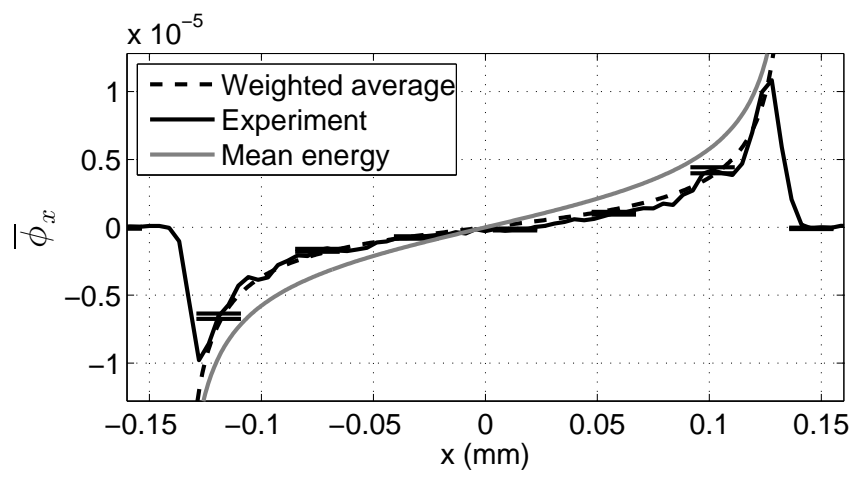

FIG. 2. Theoretical and experimental plots of $\overline{\phi_{x}}$ for the titanium wire. The "weighted average" curve was calculated using Eq. (10) whilst the "mean energy" curve was obtained using titanium's value of $\delta$ at the mean energy of the spectrum.

energy spectral components obtain a higher weighting for the thicker parts of the sample. These results are consistent with those of Fig. 4 however they do not match completely as both effective energies are slightly underestimated. We believe that this is due to an underestimation of the high energy content of the source spectrum.

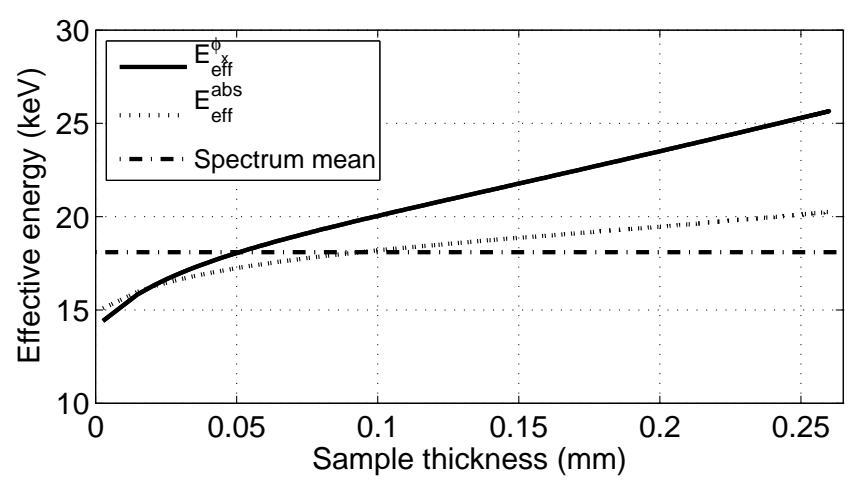

FIG. 3. Plots of how the effective energies (phase and absorption) and spectrum mean energy vary across a titanium wire of radius $130 \mu \mathrm{m}$, plotted against the thickness of the wire.

We now present some experimental results to further verify the theory which we have developed. We imaged a phantom of wires made of different materials using both a synchrotron source and a conventional source using the coded aperture technique. These experiments have been described in detail in recent publications $[6,13]$. The synchrotron results were obtained using the SYRMEP bending magnet beamline [21] at the Elettra synchrotron radiation facility in Trieste, Italy. The edge illumination set-up was realised by partially covering the Edge-On silicon microstrip single photon counting "PICASSO" detector with a fully absorbing edge [22]. Phase and absorption images were obtained for two photon energies, $20 \mathrm{keV}$ and $25 \mathrm{keV}$ and the slit used to create the single $\mathrm{x}$-ray beam were sufficiently thick so as not to exhibit any partial transmission. The experiments using a con- 
ventional source were performed using a Rigaku 007HF source operated at $35 \mathrm{kV} / 25 \mathrm{~mA}$ (mean energy approximately $18 \mathrm{keV}$ ) with a rotating Mo target. In all cases, the fibres were imaged along their length such that several profiles similar to those shown in Figs. (4) and (5) were obtained. The plots in Figs. (4) and (5) were, however, obtained by averaging profiles from the images. This introduced an error since the wires were neither straight nor aligned with the detector pixels. Thus, each profile was shifted to match up the pixels having the most positive and most negative value of $\phi_{x}$. Re-sampling or sub-pixel shifting was not performed and this is the prime reason why the error in the vicinity of the peaks in $\phi_{x}$ is large.

The first sample considered is a titanium wire of radius $130 \mu \mathrm{m}$. Comparing the plot of $\mu$ for the three source conditions in Fig. 4 shows that, in the polychromatic case, the effective energy across the sample as predicted by absorption is between 20 and $25 \mathrm{keV}$. Yet, the effective energy as predicted by the measurement of $\phi_{x}$ is greater than $25 \mathrm{keV}$. A polyetheretherketone (PEEK) wire of radius $240 \mu \mathrm{m}$ was also imaged which has much lower absorption than titanium as shown in Fig. 5. Note that $\mu$ has some artefacts at the edges of the sample due to the low absorption of PEEK. The main thing to note from these plots is that for the polychromatic case, the effective energy predicted by the measurement of $\phi_{x}$ is very close to $25 \mathrm{keV}$, lower than that predicted by the titanium sample. The effective energy predicted by the measurement of $\mu$ is closer to $20 \mathrm{keV}$ than $25 \mathrm{keV}$, which too is different from that predicted by the titanium sample. These results demonstrate that an effective energy must be defined for phase and absorption measurements independently and that these are sample specific.
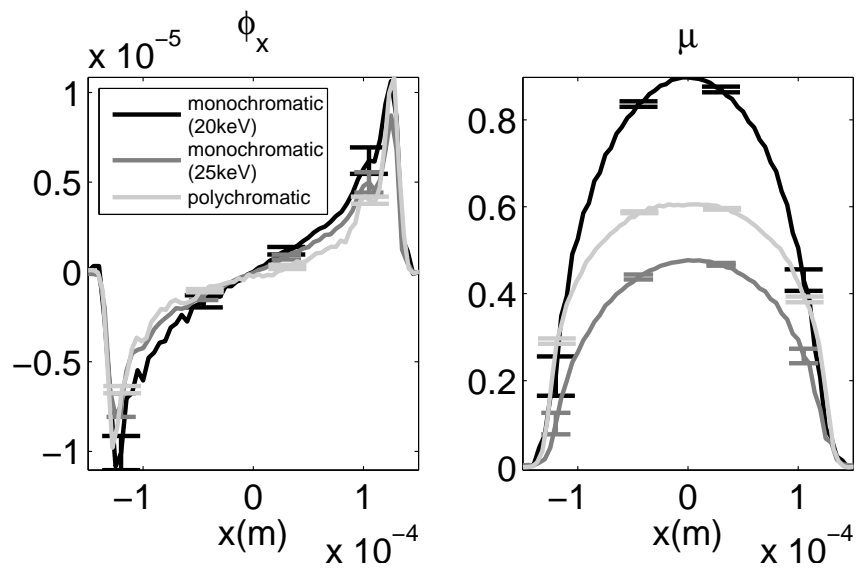

FIG. 4. Plots of $\phi_{x}$ and $\mu$ for a titanium wire of radius $130 \mu \mathrm{m}$ acquired using a synchrotron source at $20 \mathrm{keV}$ and $25 \mathrm{keV}$ respectively and using a polychromatic source. The error bars represent one standard deviation.

As a final step we present some simulated results which demonstrate how the spectrum mean energy, absorption effective energy and phase gradient effective energies dif-

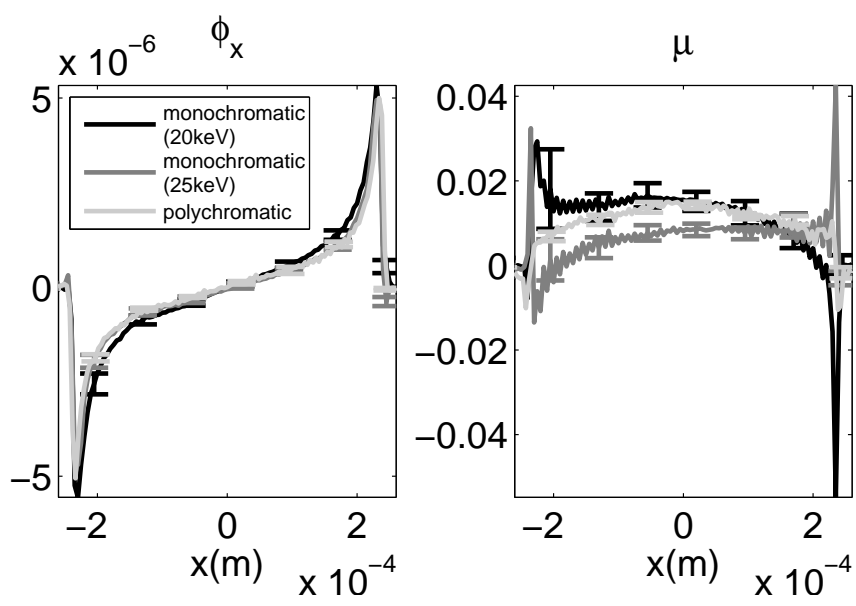

FIG. 5. Plots of $\phi_{x}$ and $\mu$ for a PEEK wire of radius $240 \mu \mathrm{m}$ acquired using a synchrotron source at $20 \mathrm{keV}$ and $25 \mathrm{keV}$ respectively and using a polychromatic source. The error bars represent one standard deviation.

fer for a material possessing low absorption. The simulation was performed using a wave optical model of the coded aperture system in a manner similar to previously reported simulations $[6,23,24]$. We have considered two cases, one with a source with low spectral width and one which employs a spectrum similar to that of the laboratory source used to generate the previously presented experimental results. In this first case we are interested in how the spectral width of a source affects the effective energies and so we have employed a spectrum having a photon fluence, $\Phi(E)$, given by

$$
\Phi(E)=\frac{1}{\sigma_{E} \sqrt{\pi}} \exp \left[-\left(\frac{E-E_{0}}{\sigma_{E}}\right)^{2}\right]
$$

where in our case we chose the center energy, $E_{0}$ to be $22.5 \mathrm{keV}$. The spectral width of the source was thus determined by $\sigma_{E}$. We modelled a coded aperture system with the same properties as described at the beginning of this section, with the exception that the thickness of the apertures $\mathrm{G}_{1}$ and $\mathrm{G}_{2}$, assumed to be equal, was varied and source photon fluence was described by Eq. (15). It is also important to point out that we have assumed that the detector is energy integrating. In order to simplify the analysis, a wedge object was modelled with its thickness changing along the direction of phase sensitivity of the coded aperture imaging system. In particular, the thickness of the wedge was described by $T(x)=x+b$ where $x$ is the spatial coordinate in the plane of $\mathrm{G}_{1}$ and in the direction of phase sensitivity and $x=0$ was assumed to be in the center of the transmitting region of the aperture. The value of $b$ thus determines the average thickness of the wedge and was varied to change the amount of absorption in the sample. We modelled a PEEK sample which has a distribution of $\delta$ and $\beta$ as plotted in Fig. 6. The simulation was performed for spectral 
widths up to $15 \mathrm{keV}$ (full width at half maximum) and aperture thicknesses up to $160 \mu \mathrm{m}$. Since the slope of the wedge was known, the value of $\bar{\delta}$ could be determined from the measured value of $\overline{\phi_{x}}$. Knowledge of the average wedge thickness allowed $\bar{\mu}$ to be calculated. From both of these quantities the effective energies $\mathrm{E}_{e f f}^{\phi_{x}}$ and $\mathrm{E}_{\text {eff }}^{\mu}$ could be found.

Fig. 7 shows plots of $\mathrm{E}_{e f f}^{\phi_{x}}, \mathrm{E}_{e f f}^{\mu}$ and spectrum mean energy for two wedge thicknesses and apertures of thickness $160 \mu \mathrm{m}$, chosen so as to result in perfectly absorbing apertures. These plots reveal the degree to which the effective energies diverge even for a relatively narrow spectrum. Consider first the thin wedge $(b=0.5 \mathrm{~mm})$. We observe that $\mathrm{E}_{e f f}^{\phi_{x}}$ decreases as the spectral width increases. This is as expected since the mean phase gradient may be found according to $\overline{\phi_{x}}=\phi_{x}\left(E_{\text {eff }}^{\phi_{x}}\right) \approx$ $\int \delta(E) \Phi(E) E \mathrm{~d} E / \int \Phi(E) E \mathrm{~d} E$ which decreases with increasing spectral width due to the $1 / E^{2}$ dependence of $\delta$. As the sample thickness increases, Eq. (14) must be used to evaluate $\overline{\phi_{x}}$ which weights higher energies more heavily due to sample absorption. The behaviour of $\mathrm{E}_{e f f}^{\mu}$ can be explained in a similar manner to $\mathrm{E}_{e f f}^{\phi_{x}}$, differing because $\mu$ has a $1 / E^{3}$ dependence. We can see from these plots that even for low absorbing materials, even modest spectral widths are sufficient to see significant variation between the respective equivalent energies.

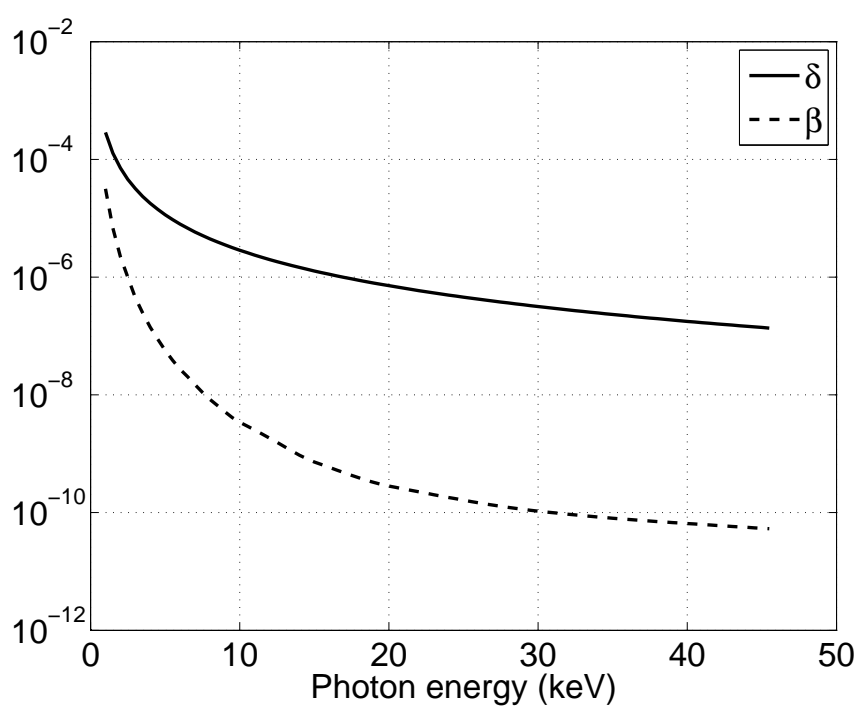

FIG. 6. The refractive index decrement and imaginary part of refractive index for PEEK [20].

In our final simulated result we consider a simulated spectrum for our laboratory source, employing a Molybdenum target, for a variety of tube voltages between $20 \mathrm{keV}$ and $42 \mathrm{keV}$. The spectra, calculated using the method of Boone et. al [19], included the effects of propagation through air and the graphite substrate upon which the apertures are mounted, although these have no effect on the observed trends. The simulated effective energies and spectrum mean energy are plotted in Fig. 8 for two different aperture thicknesses: $160 \mu \mathrm{m}$ and $20 \mu \mathrm{m}$. In the former case the apertures remain fully absorbing over the entire spectrum whilst in the latter case they are only partially absorbing at high energies. The spectrum in this case is fundamentally different to that considered previously, since the spectrum extends asymmetrically as the tube voltage increases, rather than symmetrically in the case of the Gaussian spectrum. As a result we observe all effective energies increasing with tube voltage. The lower plot which shows the example of apertures which are partially absorbing demonstrates how $\mathrm{E}_{e f f}^{\phi_{x}}$ is affected by partially transmitting apertures. In particular, we see that the weights $W_{j}^{c a}(\lambda)$ will no longer integrate to unity and in fact result in a spurious, generally lower, value of $\overline{\phi_{x}}$ being measured thus leading to higher values of $\mathrm{E}_{e f f}^{\phi_{x}}$.

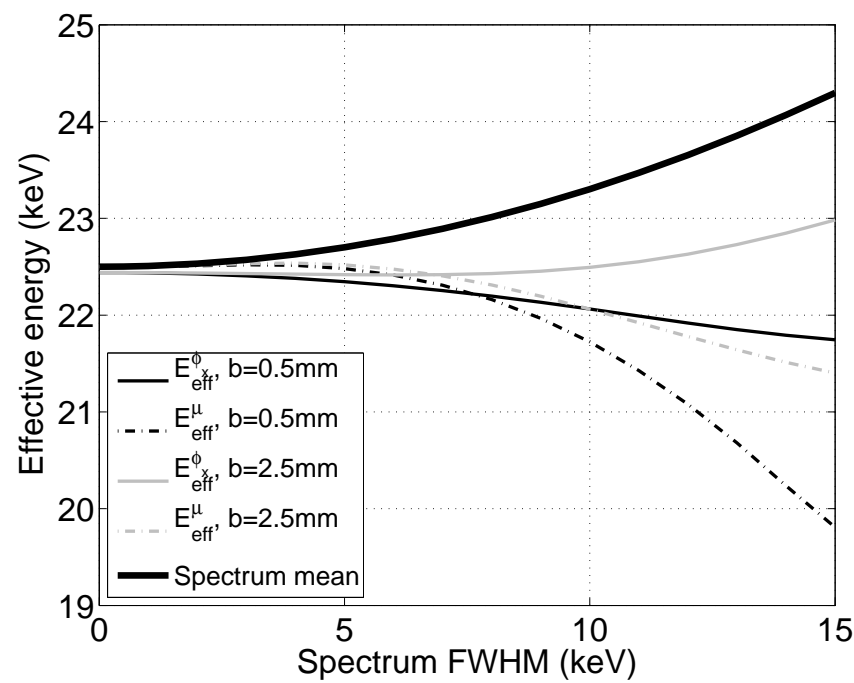

FIG. 7. Plots of $E_{\text {eff }}^{\phi_{x}}, E_{\text {eff }}^{\mu}$ and spectrum mean versus the full width at half maximum of the source spectrum defined by Eq. (15). Two different thicknesses of the PEEK wedge are considered as indicated by the legend. The apertures were assumed to be $160 \mu \mathrm{m}$ thick.

\section{CONCLUSIONS}

We conclude from this work that quantitative results obtained from polychromatic grating based XPCI systems should be interpreted with caution. In particular, it is only a special case in which effective energies relating to absorption and phase measurement and spectrum mean energy coincide, even for narrow spectra. Furthermore, it is impossible to, for example, make an absolute measurement of phase in the general case as the measured phase gradient depends on a number of factors including the optical properties of the gratings, the source spectrum and sample absorption. This also has implications for the use of the polychromatic grating XPCI technique for tomography. One possible solution to this could be 

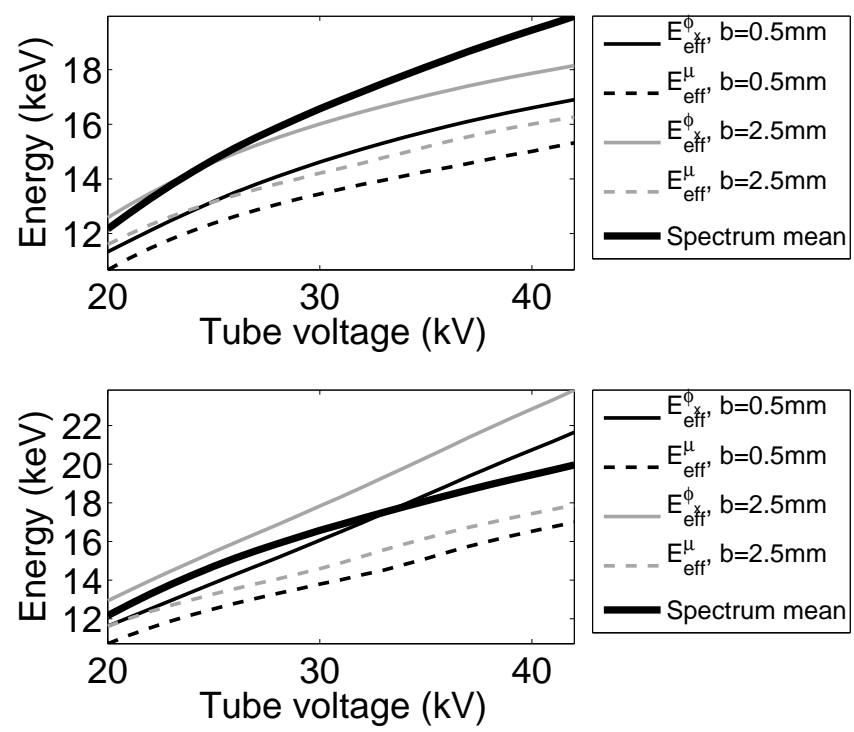

FIG. 8. Plots of $E_{\mathrm{eff}}^{\phi_{x}}, E_{\mathrm{eff}}^{\mu}$ and spectrum mean versus tube voltage, assuming a Molybdenum target, for two different thicknesses of the PEEK wedge sample and $160 \mu \mathrm{m}$ thick apertures (top) and $20 \mu \mathrm{m}$ apertures (bottom).

to try to more accurately invert Eqs. (5) to (7) by characterising the imaging system and parameterising $\delta(\lambda)$ and $\beta(\lambda)$ as done by Gureyev and Wilkins [25] and more recently Mukaide et al. [26].

We would like to thank the personnel of the Elettra synchrotron and the University of Trieste working on the SYRMEP beamline for their assistance in obtaining the synchrotron images. A.O. is supported by a Career Acceleration Fellowship (EP/G004250/1) and Challenging Engineering Grant (EP/I021884/1), both awarded by the UK Engineering and Physical Sciences Research Council. P.M. is supported by a Discovery Early Career Research Award from the Australian Research Council (DE120101331).

[1] F. Pfeiffer, T. Weitkamp, O. Bunk, and C. David, Nat. Phys. 2, 258 (2006).

[2] A. Olivo and R. Speller, Appl. Phys. Lett. 91, 074106 (2007).

[3] Z.-F. Huang, K.-J. Kang, L. Zhang, Z.-Q. Chen, F. Ding, Z.-T. Wang, and Q.-G. Fang, Phys. Rev. A 79, 013815 (2009).

[4] A. Momose, W. Yashiro, H. Kuwabara, and K. Kawabata, Jpn. J. Appl. Phys. 48, 076512 (2009).

[5] A. Tapfer, A. Bech, M. ans Velroyen, J. Meiser, J. Mohr, M. Walter, J. Schulz, B. Pauwels, P. Bruyndonckx, X. Liu, A. Sasov, and F. Pfeiffer, Proc. Natl. Acad. Sci.
U. S. A. 109, 15691 (2012).

[6] P. Munro, K. Ignatyev, R. Speller, and A. Olivo, Proc. Natl. Acad. Sci. U. S. A. 109, 13922 (2012).

[7] M. Chabior, T. Donath, C. David, O. Bunk, M. Schuster, C. Schroer, and F. Pfeiffer, Med. Phys. 38, 1189 (2011).

[8] A. Lohmann, Optik 79, 41 (1988).

[9] P. Munro, K. Ignatyev, R. Speller, and A. Olivo, Opt. Express 18, 19681 (2010).

[10] A. Olivo, F. Arfelli, G. Cantatore, R. Longo, R. Menk, S. Pani, M. Prest, P. Poropat, L. Rigon, G. Tromba, E. Vallazza, and E. Castelli, Med. Phys. 28, 1610 (2001).

[11] A. Momose, S. Kawamoto, I. Koyama, Y. Hamaishi, K. Takai, and Y. Suzuki, Jpn. J. Appl. Phys. 42, L866 (2003).

[12] T. Donath, M. Chabior, F. Pfeiffer, O. Bunk, E. Reznikova, J. Mohr, E. Hempel, S. Popescu, M. Hoheisel, M. Schuster, J. Baumann, and C. David, J. Appl. Phys. 106 (2009), 10.1063/1.3208052.

[13] P. R. Munro, L. Rigon, K. Ignatyev, F. C. Lopez, D. Dreossi, R. D. Speller, and A. Olivo21:647-661, Opt. Express 21, 647 (2013).

[14] S. Mayo, P. Miller, S. Wilkins, T. Davis, D. Gao, T. Gureyev, D. Paganin, D. Parry, A. Pogany, and A. Stevenson, Journal of Microscopy-Oxford 207, 79 (2002).

[15] T. Gureyev and S. Wilkins, J. Opt. Soc. Am. A 15, 579 (1998).

[16] D. Paganin, Coherent X-ray optics, Oxford series on synchrotron radiation (Oxford University Press, Great Clarendon Street, Oxford OX2 6DP, 2006).

[17] K. Creath, Prog. Optics XXVI, 349 (1988).

[18] E. McCullough, Medical Physics 2, 307 (1975).

[19] J. Boone, T. Fewell, and R. Jennings, Med. Phys. 24, 1863 (1997).

[20] B. Henke, E. Gullikson, and J. Davis, Atom. Data Nucl. Data 54, 181 (1993).

[21] A. Abrami, F. Arfelli, R. Barroso, A. Bergamaschi, F. Bille, P. Bregant, F. Brizzi, K. Casarin, E. Castelli, V. Chenda, L. Palma, D. Dreossi, A. Fava, R. Longo, L. Mancini, R. Menk, F. Montanari, A. Olivo, S. Pani, A. Pillon, E. Quai, S. Kaiser, L. Rigon, T. Rokvic, M. Tonutti, G. Tromba, A. Vaseotto, C. Venanzi, F. Zanconati, A. Zanetti, and F. Zanini, Nucl. Instrum. Meth. A 548, 221 (2005).

[22] F. C. Lopez, L. Rigon, R. Longo, F. Arfelli, A. Bergamaschi, R. C. Chen, D. Dreossi, B. Schmitt, E. Vallazza, and E. Castelli, Journal of Instrumentation 6, C12031 (2011), 13th International Workshop on Radiation Imaging Detectors, ETH Zurich, Zurich, Switzerland.

[23] P. Munro, K. Ignatyev, R. Speller, and A. Olivo, Opt. Express 18, 4103 (2010).

[24] P. Munro, C. Hagen, M. Szafraniec, and A. Olivo, Opt. Express 21, 11187 (2013).

[25] T. Gureyev and S. Wilkins, Opt. Commun. 147, 229 (1998).

[26] T. Mukaide, M. Watanabe, K. Takada, A. Iida, K. Fukuda, and T. Noma, Appl. Phys. Lett. 98, 111902 (2011). 